\title{
Effect of Environment Size on Curvature Redirected Walking Thresholds
}

\section{Conference Paper}

\section{Author(s):}

Nguyen, Anh; Rothacher, Yannick; Kunz, Andreas (D); Brugger, Peter; Lenggenhager, Bigna

Publication date:

2018

Permanent link:

https://doi.org/10.3929/ethz-b-000253658

Rights / license:

In Copyright - Non-Commercial Use Permitted

Originally published in:

https://doi.org/10.1109/VR.2018.8446225 


\title{
Effect of Environment Size on Curvature Redirected Walking Thresholds
}

\author{
Anh Nguyen* \\ Innovation Center Virtual Reality, ETH Zurich \\ Andreas Kunz \\ Innovation Center Virtual Reality, ETH Zurich \\ Bigna Lenggenhager \\ Neuropsychology, University Hospital Zurich \\ Peter Brugger \\ Neuropsychology, University Hospital Zurich \\ Cognitive Neuropsychology, University of Zurich
}

\begin{abstract}
Redirected walking (RDW) refers to a number of techniques that enable users to explore a virtual environment larger than the real physical space. These techniques are based on the introduction of a mismatch in rotation, translation and curvature between the virtual and real trajectories, quantified as rotational, translational and curvature gains. When these gains are applied within certain thresholds, the manipulation is unnoticeable and immersion is maintained. Existing studies on RDW thresholds reported a wide range of threshold values. These differences could be attributed to many factors such as individual differences, walking speed, or environment settings.

In this paper, we propose a study to investigate one of the environment settings that could potentially influence curvature RDW thresholds: the environment size. The detailed description of the study is also provided, where the adaptive, 2-alternative forced choice method is used to identify the detection thresholds.
\end{abstract}

\section{INTRODUCTION}

Compared to walking-in-place, or using navigation devices such as game controllers, real walking in a virtual environment (VE) has been proven to have better fidelity and immersion [12]. However, the problem with real walking arises when the VE is larger than the available physical space. A solution to this problem was proposed by Razzaque et al. [9], in which the mapping between the virtual and real trajectories is manipulated. These proposed techniques, quantified as Redirected Walking (RDW) gains, are applied on different aspects of walking such as curvature, translation, rotation. When applied within certain thresholds, these manipulations are not perceptible and immersion can be maintained. Research effort has been spent on identifying what these thresholds are, however, the thresholds found by different groups have quite high variability. For example, rotation gain thresholds found by Engel et al. [2] are 0.85 and 1.35 while they are found to be 0.67 and 1.24 by Steinicke et al. [10]; curvature gain thresholds found by Hodgson et al. [5], Nitzsche et al. [7], and Steinicke et al. [10] are also considerably different: $7.64 \mathrm{~m}, 10 \mathrm{~m}$ and $22 \mathrm{~m}$ respectively. These thresholds do not seem to be constant and have been observed to vary largely between individuals [3] and context settings [4]. Correspondingly, it is essential to understand the factors that have effects on RDW thresholds, such that an optimal RDW gain could be applied, maximizing the "compression" effect of RDW techniques while maintaining immersion.

\section{Related Work}

Neth et al. [6] conducted a study to investigate the effect of walking speed on curvature thresholds and reported that faster speed increases

*e-mail: nngoc@ethz.ch

†e-mail: yannick.rothacher@usz.ch

IEEE Virtual Reality 2018

18-22 March 2018, Reutlingen, Germany

(C) IEEE 2018 sensitivity to curvature gain. Regarding the existing work on the effects of environment's design, Steinicke et al. [11] performed a threshold identification study where they presented different visual appearances of the environment and applied textures of different visual density to the environment, consequently generating different amount of optical flow. They found that users tend to be less sensitive to RDW when the amount of optical flow is small. In another study, Paludan et al. varied the number of objects in the scene to investigate the effects of visual density on rotational gain thresholds [8] and could not establish a relationship between visual density and rotational gain thresholds. Considering that high visual density leads to higher optical flow, the two aforementioned studies seem to address the same question about the effects of optical flow on RDW thresholds but their observations are rather contradictory. Given that there are differences in the design of the environments in the two studies, it is not possible to conclude whether optical flow has an effect on RDW thresholds. While investigating the performance of different RDW algorithms in a constrained world, Hodgson et al. [4] remarked that users tended to notice the curvature gains in an aisle more than in a forest. This observation suggests that the dimension of the environment may play a role in the detection of RDW.

Given the aforementioned observations and studies, we would like to perform a study to fill a gap in understanding the effects of environment factors on RDW thresholds: the environment size. In this paper, we present our experiment design for identifying the effect of environment size on curvature RDW thresholds.

\section{USER STUdY}

\subsection{Experiment Design}

The two scenes, corresponding to two room size conditions, were designed to be as plain as possible to remove any confounding effects of optical flow. Both scenes contain a red target, which is located $7.5 \mathrm{~m}$ from the starting position of the user, four surrounding walls with simple shading and no texture on the walls. The width of the room in the one condition is $10 \mathrm{~m}$, while it is $2 \mathrm{~m}$ in the other condition. In both conditions, the room length is $10 \mathrm{~m}$. Fig. 1 shows the two scenes.

Since it has been found that walking speed affects curvature RDW thresholds [6], we control the walking speed of users by asking them to follow certain step sound. The step sound frequency is generated by equation provided by Dean [1]. Using the 2-alternative forced choice method, in each trial each user walks to the target two times. In only one of the two walks, a curvature gain is applied and the order is randomized between trials. The next value of curvature gain to be tested is calculated by a Bayesian adaptive method called "QUEST" by Watson and Pelli [13].

\subsection{Experiment Setup}

The experiment setup consists of an Oculus DK2 with a built-in SMI eye tracker and an Intersense IS-1200 inside-out optical tracking system mounted on top, providing 6-DOF positional tracking at $180 \mathrm{~Hz}$. A cover is added in front of the headset to prevent users from 


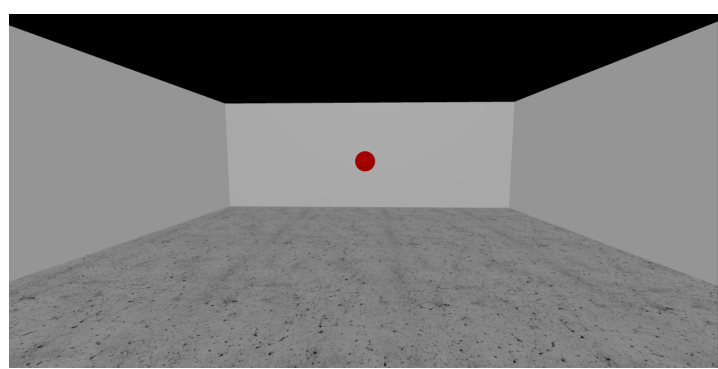

(a) $10 \mathrm{~m}$ wide room

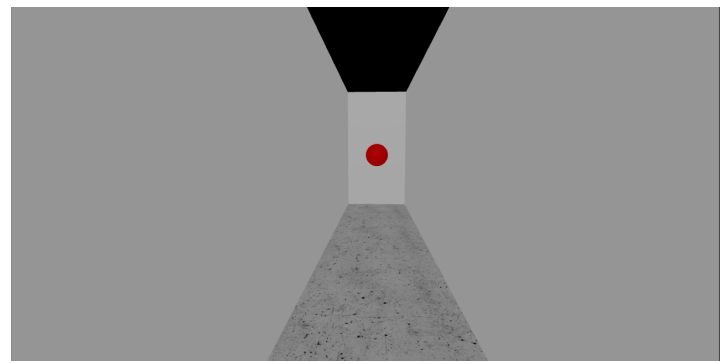

(b) $2 \mathrm{~m}$ wide room

Figure 1: Types of scenes

seeing the floor. The scenes are optimally designed in Unity to run at the HMDs maximum frame rate $75 \mathrm{~Hz}$. The whole setup is powered by a backpack-mounted notebook. The available tracking space is $12 \mathrm{~m} \times 6 \mathrm{~m}$. The users also wear noise cancelling headphones, where the step regulating rhythms are played.

\section{Results}

60 subjects (30 men, right-handed, with normal or corrected vision) were recruited from the university market place and paid $15 \mathrm{CHF} /$ hour for their participation. 30 participants were exposed to the $2 \mathrm{~m}$ wide room condition and the other 30 participants the $10 \mathrm{~m}$ wide room condition (same number of women and men tested with each room size condition). To investigate the effect of room size condition (between-subject variable), we fitted a linear mixed model which includes room size as a fixed factor and subjects as a random factor. The full mixed-effect model therefore becomes: Threshold $\sim 1+$ RoomSize $+(1 \mid$ Subject $)$. Results did not show a significant effect of room size on curvature RDW thresholds $(p>0.05)$.

\section{Discussion}

It was surprising to find out that room's dimension does not significantly affect curvature gain thresholds. This contradicts with the observations by Hodgson et al. [5] that their subjects recognized curvature gains more in the aisle compared to an open room. One possible explanation for this finding could be that it is not the room dimension, but the amount of optical flow generated by the scene or the combination of room dimension and optical flow that affect curvature gain thresholds. Since we had no texture on the walls, the amount of optical flow generated in both cases was mostly similar. It could still be possible that there is a small effect that can not be recovered in a between-subject design since there is a high variability in individual thresholds. A within-subject design where each subjects thresholds are measured in both room size conditions would have been more suitable.

\section{Conclusion}

In this paper, we have proposed a study to investigate the effect of environment size on curvature gain thresholds. We found that environment size does not have a significant effect on curvature gain thresholds possibly due to the lack of optical flow. Further study is required to identify which other factors of the environment influence RDW thresholds such that a set of guidelines about how much curvature gain should be applied in a given virtual environment could be obtained.

\section{ACKNOWLEDGMENTS}

This work was supported by a grant from the Swiss National Science Foundation. The experiment was approved by the cantonal ethics committee.

\section{REFERENCES}

[1] G. A. Dean. An analysis of the energy expenditure in level and grade walking. Ergonomics, 8(1):31-47, 1965. doi: 10.1080/ 00140136508930772

[2] D. Engel, C. Curio, L. Tcheang, B. Mohler, and H. H. Bülthoff. A psychophysically calibrated controller for navigating through large environments in a limited free-walking space. In Proceedings of the 2008 ACM Symposium on Virtual Reality Software and Technology, VRST '08, pp. 157-164. ACM, New York, NY, USA, 2008. doi: 10. $1145 / 1450579.1450612$

[3] T. Grechkin, J. Thomas, M. Azmandian, M. Bolas, and E. Suma. Revisiting detection thresholds for redirected walking: Combining translation and curvature gains. In Proceedings of the ACM Symposium on Applied Perception, SAP '16, pp. 113-120. ACM, New York, NY, USA, 2016. doi: 10.1145/2931002.2931018

[4] E. Hodgson, E. Bachmann, and T. Thrash. Performance of redirected walking algorithms in a constrained virtual world. IEEE Transactions on Visualization and Computer Graphics, 20(4):579-587, Apr. 2014. doi: 10.1109/TVCG.2014.34

[5] E. Hodgson, E. Bachmann, and D. Waller. Redirected walking to explore virtual environments: Assessing the potential for spatial interference. ACM Trans. Appl. Percept., 8(4):22:1-22:22, Dec. 2008. doi: 10.1145/2043603.2043604

[6] C. T. Neth, J. L. Souman, D. Engel, U. Kloos, H. H. Bülthoff, and B. J. Mohler. Velocity-dependent dynamic curvature gain for redirected walking. In 2011 IEEE Virtual Reality Conference, pp. 151-158. IEEE, New York, NY, USA, March 2011. doi: 10.1109/VR.2011.5759454

[7] N. Nitzsche, U. D. Hanebeck, and G. Schmidt. Motion compression for telepresent walking in large target environments. Presence, 13(1):4460, Feb 2004. doi: 10.1162/105474604774048225

[8] A. Paludan, J. Elbaek, M. Mortensen, M. Zobbe, N. C. Nilsson, R. Nordahl, L. Reng, and S. Serafin. Disguising rotational gain for redirected walking in virtual reality: Effect of visual density. In Virtual Reality (VR), 2016 IEEE, pp. 259-260. IEEE, 2016.

[9] S. Razzaque, Z. Kohn, and M. C. Whitton. Redirected Walking. In Eurographics 2001 - Short Presentations, pp. 1-6. Eurographics Association, Geneva, Switzerland, 2001. doi: 10.2312/egs. 20011036

[10] F. Steinicke, G. Bruder, J. Jerald, H. Frenz, and M. Lappe. Estimation of detection thresholds for redirected walking techniques. IEEE Transactions on Visualization and Computer Graphics, 16(1):17-27, Jan. 2010. doi: 10.1109/TVCG.2009.62

[11] F. Steinicke, G. Bruder, T. Ropinski, and K. H. Hinrichs. Moving towards generally applicable redirected walking. In Proceedings of the Virtual Reality International Conference (VRIC), pp. 15-24. IEEE Press, 2008.

[12] M. Usoh, K. Arthur, M. C. Whitton, R. Bastos, A. Steed, M. Slater, and F. P. Brooks, Jr. Walking > walking-in-place $>$ flying, in virtual environments. In Proceedings of the 26th Annual Conference on Computer Graphics and Interactive Techniques, SIGGRAPH '99, pp. 359-364. ACM Press/Addison-Wesley Publishing Co., New York, NY, USA, 1999. doi: $10.1145 / 311535.311589$

[13] A. B. Watson and D. G. Pelli. Quest: A bayesian adaptive psychometric method. Perception \& Psychophysics, 33(2):113-120, Mar 1983. doi: $10.3758 / \mathrm{BF} 03202828$ 